\title{
Effect of Bicuspid Aortic Valve Cusp Fusion on Aorta Wall Shear Stress: Preliminary Computational Assessment and Implication for Aortic Dilation
}

\author{
Kai Cao, Philippe Sucosky* \\ Department of Aerospace and Mechanical Engineering, University of Notre Dame, Notre Dame, USA \\ Email: ${ }^{*}$ philippe.sucosky@nd.edu
}

Received 31 March 2015; accepted 23 May 2015; published 26 May 2015

Copyright (C) 2015 by authors and Scientific Research Publishing Inc.

This work is licensed under the Creative Commons Attribution International License (CC BY). http://creativecommons.org/licenses/by/4.0/

(c) (i) Open Access

\section{Abstract}

The bicuspid aortic valve (BAV) is a major congenital valvular abnormality and is associated with a high prevalence of aortic dilation, whose expression depends on the type of leaflet fusion. Although BAV hemodynamics is considered a potential pathogenic contributor, the impact of BAV fusion on ascending aorta (AA) wall shear stress (WSS) remains largely unknown. A fluid-structure interaction approach was implemented to predict the hemodynamics and WSS characteristics in realistic AA models subjected to the flow of a normal tricuspid aortic valve (TAV) and three BAV morphotypes (left-right coronary cusp fusion (LR), right-non coronary cusp fusion (RN) and nonleft coronary cusp fusion (NL)). TAV flow conditions subjected the proximal and middle AA to a streamlined flow typical of flows in bends, while BAV flow conditions generated increased flow helicity. The LR-BAV orifice jet generated flow abnormalities primarily in the proximal AA, which were marked by a uniform WSS overload along the wall circumference and contrasted WSS directionalities on the wall convexity and concavity. Flow abnormalities generated by the RN-BAV and NL-BAV inlet flow conditions were more diffuse and consisted of WSS overloads in the convexity of the proximal and middle AA and contrasted WSS directionalities in the anterior and posterior wall regions. This study demonstrates the impact of the BAV morphotype on AA hemodynamics and provides quantitative evidence for the existence of WSS abnormalities in aortic wall regions prone to dilation.

\section{Keywords}

Bicuspid Aortic Valve (BAV), Aorta, Aortopathy, Hemodynamics, Fluid-Structure Interaction (FSI)

\footnotetext{
${ }^{*}$ Corresponding author.

How to cite this paper: Cao, K. and Sucosky, P. (2015) Effect of Bicuspid Aortic Valve Cusp Fusion on Aorta Wall Shear Stress: Preliminary Computational Assessment and Implication for Aortic Dilation. World Journal of Cardiovascular Diseases, 5, 129-140. http://dx.doi.org/10.4236/wjcd.2015.56016
} 


\section{Introduction}

The congenital bicuspid aortic valve (BAV) is a valvular defect present in $1 \%-2 \%$ of the general population [1]. While the BAV is characterized by the presence of two leaflets, it covers different morphologic phenotypes [2]. The most prevalent type-I morphology features two cusps of unequal size (resulting from the fusion of two of the three leaflets) and a fibrous raphe at the location of congenital fusion. While $71 \%$ of type-I BAVs result from the fusion between the left- and right-coronary leaflets (LR subtype), 15\% feature right- and non-coronary cusp fusion (RN subtype) and 3\% present with non- and left-coronary cusp fusion (NL subtype) [3]. As compared with tricuspid aortic valve (TAV) patients, BAV patients have a much higher risk of developing secondary aortopathies such as aortic dilation [4].

While BAV aortopathy has been historically linked to a genetic predisposition, the abnormal BAV hemodynamics has emerged as a potential coincident etiology [5] [6]. Phase-contrast magnetic resonance imaging (PC MRI) and echocardiographic measurements in BAV patients [7]-[10], as well as in vitro particle-image velocimetry measurements [11]-[13] and in silico simulations in idealized ascending aorta (AA) geometries [14]-[17] have revealed the existence of abnormal helical flow patterns and fluid wall shear stress (WSS) overloads in AA regions vulnerable to aortic medial degeneration. In addition, the particular leaflet fusion type affects the expression of dilation and thinning of the AA downstream of a BAV. While the LR subtype is primarily associated with dilation of the aortic root and the AA convexity, dilation patterns downstream of RN-BAVs localize to the tubular AA and sometimes extend to the transverse aortic arch [18] [19].

Collectively, those results provide compelling evidence for a possible role of hemodynamics in the pathogenesis of BAV aortopathy. However, the validation of this hemodynamic pathway requires the characterization of the flow patterns generated by each BAV subtype and their impacts on AA wall biology. A first attempt at establishing this causality was successfully made in our laboratory through the implementation of a combined computational and ex vivo approach, which demonstrated the susceptibility of LR-BAV hemodynamic stresses to focally mediate aortic medial degeneration [17]. To complement this initial effort, this study aims at providing a preliminary quantitative assessment of the impact of different type-I BAV cusp fusions (i.e., LR, RN and NL) on AA hemodynamics and WSS using three-dimensional (3D) fluid-structure interaction (FSI) modeling.

\section{Methods}

\subsection{Aorta Geometry and Constitutive Models}

The 3D aorta geometry was based on a human anatomy reconstructed from a series of histological computed tomography images, as previously described [17]. The final geometry included the AA, the transverse aortic arch (along with the proximal segments of the brachiocephalic (BCA), left common carotid (LCCA) and left subclavian (LSCA) arteries), and the thoracic descending aorta (Figure 1). The aortic wall was approximated as a linear, elastic and isotropic material (elastic modulus: $2 \mathrm{MPa}$; Poisson's ratio: 0.45) and blood was modeled as an incompressible, homogeneous and Newtonian fluid (density: $1050 \mathrm{~kg} / \mathrm{m}^{3}$; dynamic viscosity: 0.0035 Pa.s).

\subsection{Boundary Conditions}

Four simulations were performed by imposing four different 3D pulsatile and spatially dependent flow velocity profiles (i.e., TAV, LR-BAV, RN-BAV and NL-BAV) at the inlet section of the AA. The TAV and LR-BAV velocity profiles were generated by extrapolating the pulsatile velocity profiles predicted at the sinotubular junction by our previous 2D TAV and BAV FSI models [14]. Specifically, based on the near angular and near planar symmetry of the TAV and BAV anatomy, respectively, the 3D TAV velocity profile was created by revolving the 2D TAV velocity profile about the inlet axis, while the 3D LR-BAV velocity profile was obtained by extrapolating the 2D BAV velocity profile in the transverse direction as a parabolic profile satisfying the no-slip condition at the wall. The resulting velocity profiles were then scaled to match physiologic TAV and BAV mean flow rates of 4.3 and $3.5 \mathrm{~L} / \mathrm{min}$, respectively [8]. The specifics of this extrapolation method have been previously published and validated [17] and the resulting 3D velocity profiles have been shown to generate similar aortic flow patterns as those observed using PCMRI downstream of TAVs and LR-BAVs [8] [20]. The RN-BAV and NL-BAV velocity profiles were obtained by rotating the LR-BAV velocity profile by $120^{\circ}$ and $240^{\circ}$, respectively, in order to reflect the different cusp fusion patterns (Figure 2). The boundary conditions at the four outlets of the aorta model (BCA, LCCA, LSCA and descending aorta) consisted of physiologic pressure waveforms 


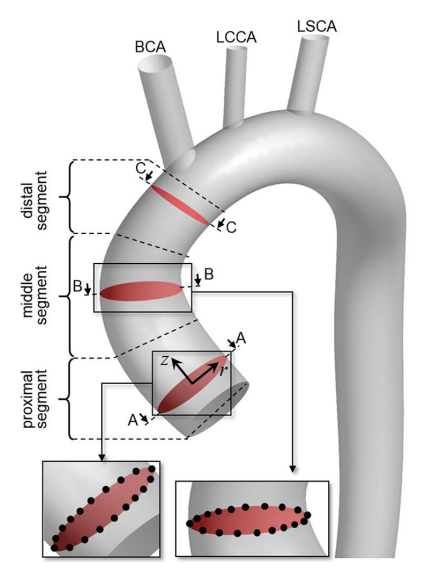

Figure 1. Aorta geometry (A-A: proximal section, B-B: middle section, C-C: distal section, insets: longitudinal WSS characterization points along the wall circumference in the proximal and middle sections).
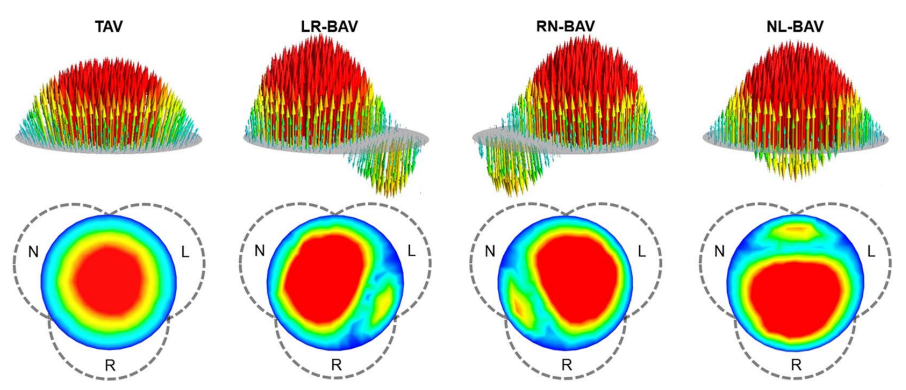

Figure 2. Aorta inlet flow conditions: peak-systolic 3D inlet velocity profiles (top: velocity vector field; bottom: transverse view of the velocity contour field and sinus wall outline; L: left-coronary sinus; R: right-coronary sinus; N: non-coronary sinus).

obtained from the literature [21]. All waveforms reflected a cardiac period of $0.86 \mathrm{~s}$ (i.e., heart rate of 70 beats per minute). The aortic wall was constrained in the axial direction at the inlet and outlet sections but was allowed to move in the radial and circumferential directions at all locations. A linear elastic support condition with a foundation stiffness of $75 \mathrm{mmHg} / \mathrm{mm}$ [22] was prescribed on the outer aortic wall to account for the damping effect generated by the surrounding tissues on the aorta.

\subsection{Computational Approach}

The system coupling module of the commercial software ANSYS (ANSYS Inc.) was used to model the interactions between the aortic wall and blood flow. The governing equations consisted of the momentum and continuity equations in their arbitrary Lagrangian Eulerian (ALE) forms for the fluid domain (i.e., blood) and the momentum equation for the structure (i.e., aortic wall), as previously described [23]. Those equations were solved along with three coupling conditions enforcing continuity of displacements, continuity of velocities (no-slip) and compatibility of traction at the fluid-structure interface. The Navier-Stokes and continuity equations were solved using a finite volume method in ANSYS Fluent. The implicit flow solver used a second-order upwind spatial discretization scheme and a second-order temporal discretization scheme. The convergence criterion for the continuity and momentum equations was set at $10^{-6}$. The structural solver employed an implicit direct displacement-based finite element method in ANSYS Mechanical. The system of equations was solved using the sparse direct solver and the equilibrium equations were solved by the Newton-Raphson method. The convergence criterion was determined by multiplying the reference value determined by ANSYS based on external forces by a tolerance of $0.5 \%$. All simulations were carried out over three cardiac cycles to achieve temporal convergence and all results were extracted from the last cycle. The aortic wall and lumen were meshed with quad/tri elements. 
A mesh sensitivity analysis was performed to determine an appropriate mesh density for the fluid domain. This analysis was conducted on the TAV AA geometry and consisted of quantifying the convergence of the timeaveraged WSS predicted over the entire aortic wall under increasing mesh density. The initial coarse mesh was based on a maximum element size of $1.3 \mathrm{~mm}$ and consisted of 194,821 elements. The progressive decrease of the maximum element size by $8 \%, 23 \%, 38 \%$ and $54 \%$ produced a $12 \%, 20 \%, 22 \%$ and $22 \%$ increase, respectively, in the time-averaged WSS predicted over the entire aortic wall relative to the value obtained with the initial mesh. The second mesh refinement, which generated less than $2 \%$ change in time-averaged WSS relative to the finest grid tested, was considered sufficiently resolved to capture the flow in all AA models. The final computational grid consisted of 98,625 elements in the structural domain and 368,149 in the fluid domain, which permitted to achieve a spatial resolution of $100 \mu \mathrm{m}$ in the fluid domain near the aortic wall. Each simulation required 30 hours of computing time on a Dell Precision T7500 workstation (dual quad-core $3.20 \mathrm{GHz}$ Intel Xeon processors, 48 GB DDR3 SDRAM).

\subsection{Flow and WSS Characterization}

Aorta hemodynamics was assessed in terms of streamline, velocity and WSS fields captured during the acceleration phase $(t=180 \mathrm{~ms})$, at peak systole $(t=220 \mathrm{~ms})$ and during the deceleration phase $(t=340 \mathrm{~ms})$. The spatial variations of the velocity profile along the streamwise direction were investigated by characterizing the local velocity contour field over three cross-sections located $10 \mathrm{~mm}$ (proximal section), $35 \mathrm{~mm}$ (middle section) and 55 mm (distal section) downstream of the AA inlet (see Figure 1). In addition, the angular distribution of the longitudinal WSS component $\tau_{r z}$ (i.e., component acting in the direction of the main flow) was investigated in the proximal and middle AA sections at 18 equi-angularly spaced points $\left(20^{\circ}\right.$ increment) along the wall circumference (see insets in Figure 1). At each point, the longitudinal WSS was characterized in terms of its peak-systolic value $\tau_{r z ~ S Y S}(\theta)$ as well as its temporal characteristics quantified in terms of temporal shear magnitude (TSM),

$$
\operatorname{TSM}(\theta)=\frac{1}{T} \int_{0}^{T}\left|\tau_{r z}(\theta, t)\right| \mathrm{d} t,
$$

and oscillatory shear index (OSI),

$$
\operatorname{OSI}(\theta)=\frac{1}{2}\left[1-\left(\left|\int_{0}^{T} \tau_{r z}(\theta, t) \mathrm{d} t\right| / \int_{0}^{T}\left|\tau_{r z}(\theta, t)\right| \mathrm{d} t\right)\right]
$$

respectively, where $\tau_{r z}(\theta, t)$ represents the local longitudinal WSS on the wall circumference at time $t$ and $T$ is the cardiac period. Those WSS characteristics were also investigated in the posterior convexity, posterior concavity, anterior convexity and anterior concavity of the wall by calculating the average peak-systolic WSS, TSM and OSI over each quadrant.

\section{Results}

\subsection{General Flow Characteristics}

During the acceleration phase (Figure 3(a)), the streamlines remain essentially parallel to the aortic wall in all AA models. The velocity profile predicted in the proximal section of the TAV, RN-BAV and NL-BAV AAs strongly reflects the characteristics of the flow prescribed at the AA inlet. In that region, the TAV AA velocity remains axisymmetric, while the RN-BAV and NL-BAV AA velocities exhibit the same skewness as that imposed at the AA inlet. In contrast, the initial skewness imposed at the LR-BAV AA inlet is less marked in the proximal section. In all models, the flow development from the proximal to the middle AA section exhibits the typical skewness of flows in the entrance region of curved pipes, marked by increased inertia near the wall concavity and low inertia near the convexity. Between the middle and distal sections, the velocity distribution becomes essentially axisymmetric and is less dependent on the valve anatomy. At peak systole (Figure 3(b)), the increase in flow momentum generates significant flow disturbances. In the TAV AA, the streamlines remain essentially parallel to the aortic wall but a few peripheral streamlines wrap around the main flow in the counterclockwise direction. The increase in flow momentum generates more disturbance in the BAV AAs, as suggested by the extensive wrapping of the low-velocity peripheral streamlines around the main flow and their backward deflection toward the valve. The combined effects of the wall curvature and increased flow helicity generate 


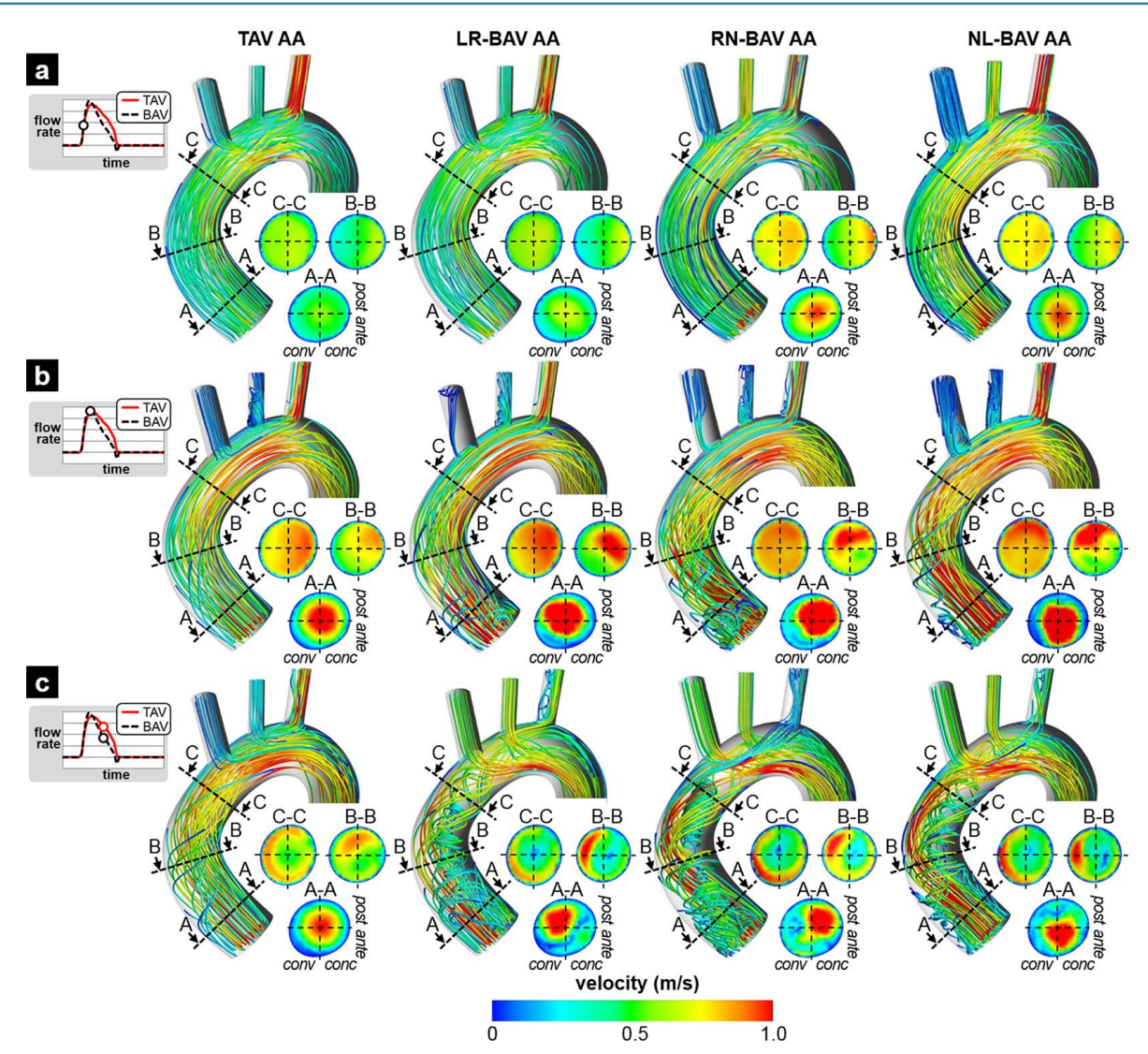

Figure 3. Velocity and streamline fields: (a) Acceleration phase, (b) Peak systole, and (c) Deceleration phase (A-A: proximal section, B-B: middle section, C-C: distal section, post: posterior, ante: anterior, conv: convexity, conc: concavity).

complex alterations of the jet skewness as the flow develops along the streamwise direction. In the LR-BAV AA, the high velocity region migrates from the posterior convexity quadrant in the proximal section toward the posterior concavity quadrant in the middle and distal sections. In contrast, the initial skewness of the flow toward the posterior concavity and the anterior region of the proximal RN-BAV AA and NL-BAV AA, respectively, relocates to the posterior convexity in the middle section and to the posterior concavity in the distal section. During the deceleration phase (Figure 3(c)), the decrease in flow momentum is accompanied by an overall increase in flow helicity in all AA models, as suggested by the recruitment of additional streamlines in counterclockwise swirling motion and their penetration into the core of the flow. While this phenomenon remains moderate and localized between the proximal and middle sections of the TAV AA, it is more pronounced and extends to the distal section in all BAV AAs. As a result, while the valve anatomy strongly affects the degree of flow asymmetry in the proximal AA, it has little effect on the flow in the distal AA.

\subsection{Global WSS Characteristics}

During the acceleration phase (Figure 4(a)), the WSS is low and relatively uniform in the proximal and middle segments of the TAV and LR-BAV AAs and increases progressively downstream to attain its maximum in the transverse aortic arch. While the WSS levels predicted in the proximal RN-BAV and NL-BAV AAs are also moderate, those morphotypes generate large spatial velocity gradients in the middle AA subjecting the wall concavity to WSS overload. The WSS remains high in the distal segment of the RN-BAV and NL-BAV AAs. At peak systole (Figure 4(b)), the TAV and LR-BAV subject the proximal AA to essentially similar WSS distributions with high magnitude on the wall convexity and concavity. In contrast, the RN-BAV imposes WSS overloads on the anterior convexity and posterior concavity regions of the proximal AA, while the NL-BAV generates WSS overloads on the anterior concavity and posterior convexity regions. In the middle and distal AA 


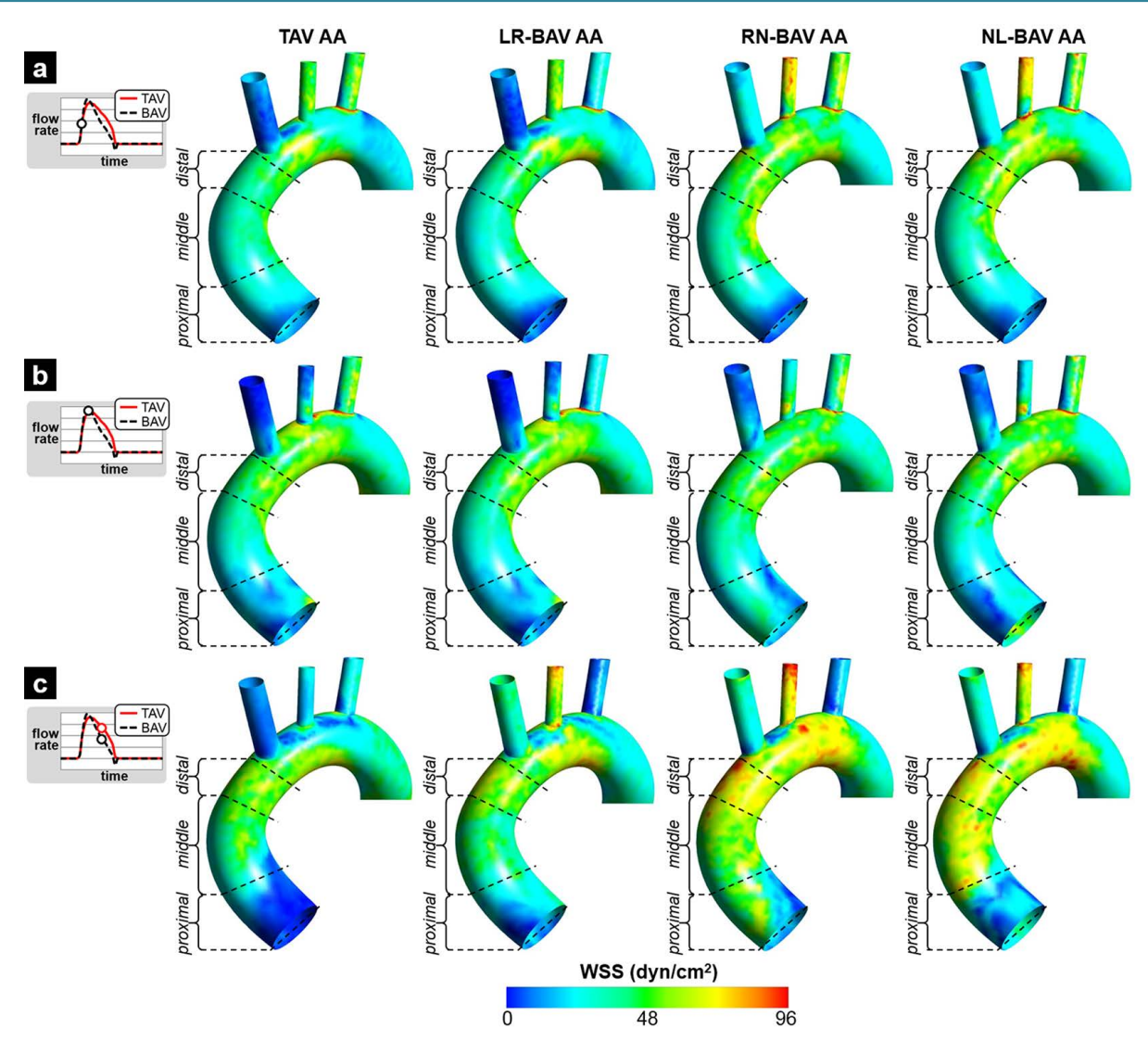

Figure 4. Total WSS fields: (a) Acceleration phase, (b) Peak systole, and (c) Deceleration phase.

segments, the wall regions experiencing high WSS magnitudes localize to the concavity, regardless of the valve anatomy. During the deceleration phase (Figure 4(c)), the decrease in flow momentum results in a net reduction in WSS in the proximal TAV AA, while the increased flow helicity detected in the middle and distal TAV AA generates a WSS overload in those regions. The intense flow helicity predicted in all three BAV AAs results in a marked increase in WSS. While those high WSS levels remain localized to the distal segment of the LR-BAV AA, they diffuse over the entire wall of the RN-BAV and NL-BAV AAs.

\subsection{Peak-Systolic Longitudinal WSS Distribution}

In all models, the peak-systolic longitudinal WSS captured in the proximal AA (Figure 5(a)) reflects the characteristics of the inlet velocity profile. $\tau_{r z}$ SYS is essentially uniform over the entire circumference of the TAV AA and more asymmetric in all BAV AAs. This asymmetry subjects the BAV AAs to a positive stress overload in the region impinged by the valvular jet and a negative stress in the diametrically opposite region. Those characteristics are particularly evident in the LR-BAV AA (mean convexity $\tau_{r z}$ sYs: $+13 \mathrm{dyn} / \mathrm{cm}^{2}$; mean concavity $\tau_{r z S Y S}:-9.2 \mathrm{dyn} / \mathrm{cm}^{2}$ ). The backward deflection of the low-velocity peripheral streamlines observed in the proximal RN-BAV and NL-BAV AAs results in a negative peak-systolic longitudinal WSS in the anterior convexity of the RN-BAV AA $\left(\tau_{r z}\right.$ SYS $\left.=-32.5 \mathrm{dyn} / \mathrm{cm}^{2}\right)$ and in the posterior convexity of the NL-BAV AA $\left(\tau_{r z}\right.$ SYS $=-29.5$ $\mathrm{dyn} / \mathrm{cm}^{2}$ ). The flow development from the proximal to the middle AA section is accompanied by an overall increase in longitudinal WSS magnitude (Figure 5(b)). At the same time, the curvature of the aortic wall tends to eliminate the effects of the initial discrepancies in jet skewness imposed at the AA inlet and to normalize the longitudinal WSS distributions among all models. As a result, the longitudinal WSS distributions in the TAV, LR-BAV and RN-BAV AAs exhibit similar patterns with a stress overload in the posterior concavity quadrant of the wall $\left(\tau_{r z \text { SYS }}>44.6 \mathrm{dyn} / \mathrm{cm}^{2}\right)$ and moderate stress levels in the other quadrants $\left(\tau_{r z \text { SYS }}<36.2 \mathrm{dyn} / \mathrm{cm}^{2}\right)$. The NL-BAV AA exhibits more contrasted peak-systolic WSS distributions in the posterior and anterior regions of the wall (mean $\tau_{r z \text { SYs: }} 43.3$ and $19.7 \mathrm{dyn} / \mathrm{cm}^{2}$, respectively). 


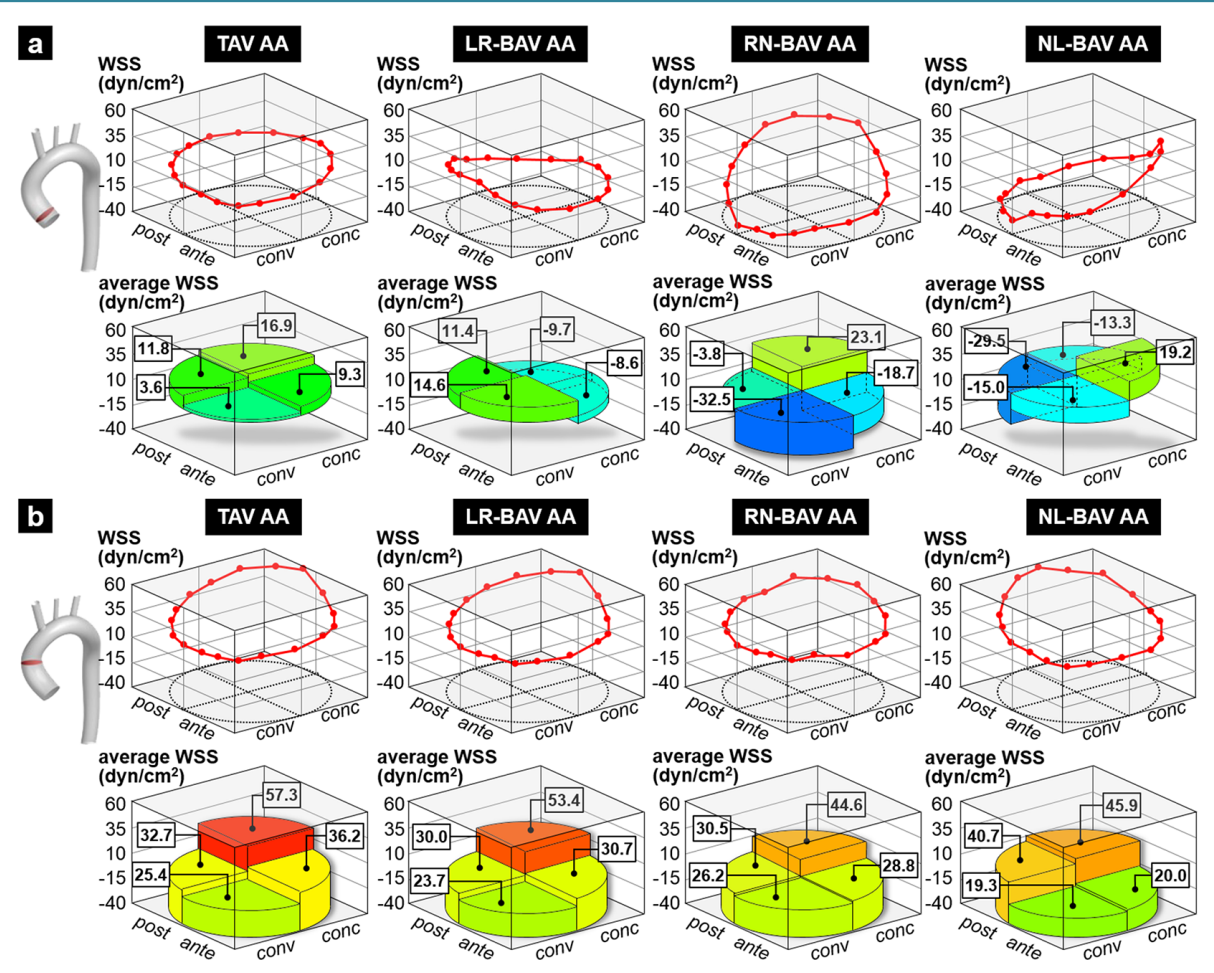

Figure 5. Local and quadrant-averaged longitudinal WSS distributions at peak systole in (a) the proximal section, and (b) the middle section of each model (post: posterior, ante: anterior, conv: convexity, conc: concavity).

\subsection{TSM Distribution}

In the proximal AA (Figure 6(a)), the TAV generates a relatively uniform TSM distribution along the wall circumference. The wall concavity and convexity are exposed to mild WSS levels (mean TSM: 5.3 and 7.0 $\mathrm{dyn} / \mathrm{cm}^{2}$, respectively). The BAV AAs exhibit a higher degree of TSM variability along the wall circumference and the extrema locations depend on the particular BAV fusion type. In addition, all BAVs subject the proximal AA to a stress overload relative to the TAV. While this overload affects the entire LR-BAV AA wall ( $38 \%$ and $22 \%$ TSM increase in the convexity and concavity, respectively, vs. TAV AA), it localizes only to the anterior convexity of the RN-BAV AA (191\% TSM increase vs. TAV AA) and to the posterior convexity of the NLBAV AA (117\% TSM increase vs. TAV AA). In the middle AA section (Figure 6(b)), the flow development occurring along the wall curvature increases the overall TSM levels in all models (58\%, 30\%, 27\% and 26\% increase vs. proximal section in TAV-AA, LR-BAV AA, RN-BAV AA and NL-BAV AA, respectively). The wall curvature effect tends to eliminate the TSM discrepancies observed in the proximal TAV and LR-BAV AAs by making the distributions more equally asymmetric (high TSM in the posterior region, low TSM in the anterior region). Similarly, the RN-BAV and NL-BAV generate essentially similar and asymmetric TSM distributions in the middle AA section (high TSM in the convexity region, low TSM in the concavity region).

\subsection{OSI Distribution}

In the proximal AA section (Figure 7(a)), the TAV generates a bidirectional longitudinal WSS (OSI $>0.30$ ), which becomes nearly purely oscillatory in the posterior wall region (OSI > 0.43). The OSI distributions predicted in all BAV AAs consistently exhibit two peaks, whose locations depend on the BAV morphotype. In the LR-BAV AA, the longitudinal WSS is unidirectional (OSI < 0.05) on the wall convexity (i.e., region impinged by the valve jet) and more bidirectional (OSI $>0.21$ ) on the concavity. The RN-BAV subjects the four quadrants of the proximal AA wall to lesser OSI variations $(0.16<$ OSI $<0.23)$. Lastly, the NL-BAV generates a contrasted WSS environment characterized by unidirectionality (OSI $<0.09$ ) in the posterior convexity and anterior concavity and moderate bidirectionality $(0.18<$ OSI $<0.25)$ in the posterior concavity and anterior convexity of 

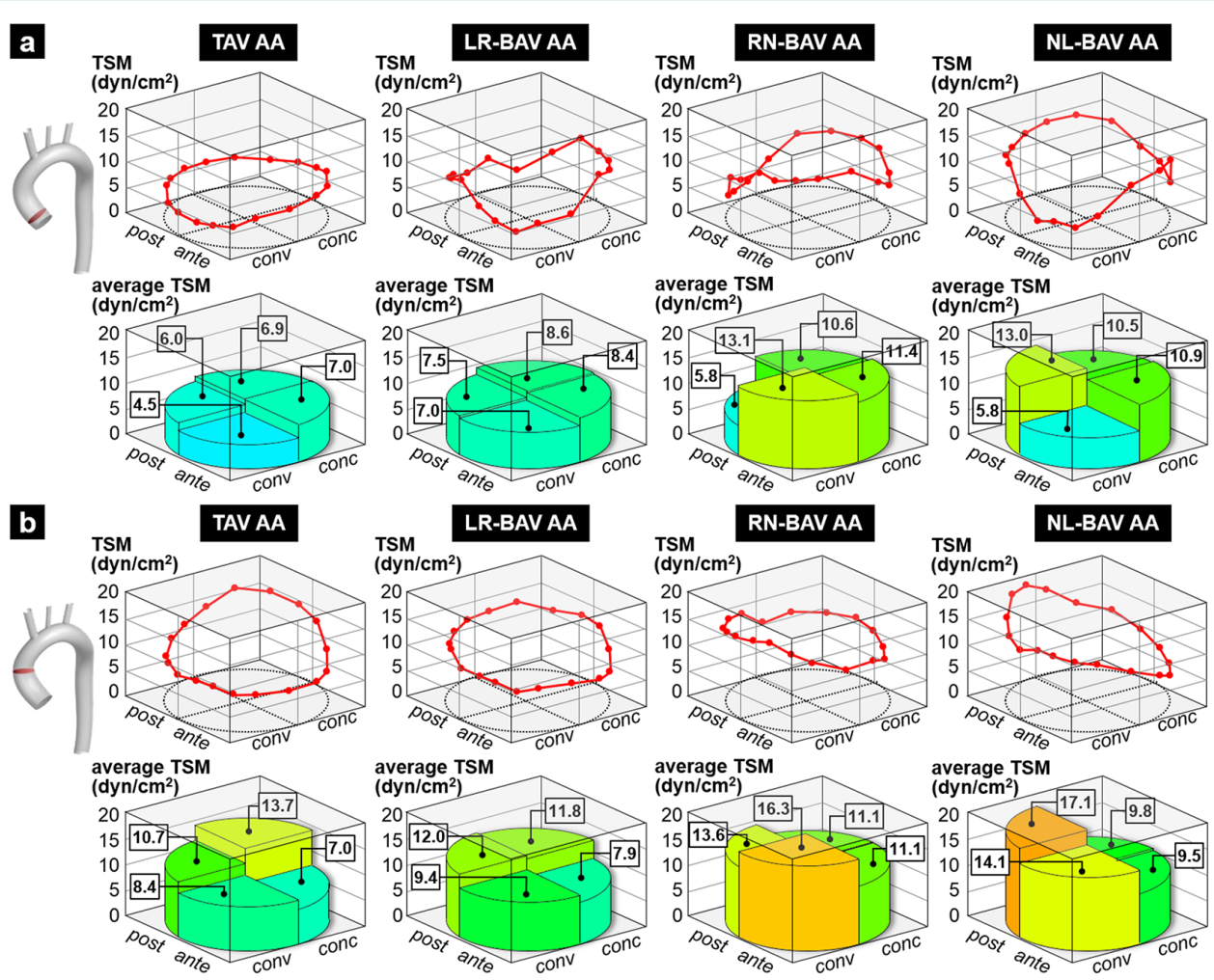

Figure 6. Local and quadrant-averaged TSM distributions in (a) the proximal section, and (b) the middle section of each model (post: posterior, ante: anterior, conv: convexity, conc: concavity).

$\mathbf{a}$

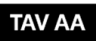

LR-BAV AA

RN-BAV AA

NL-BAV AA
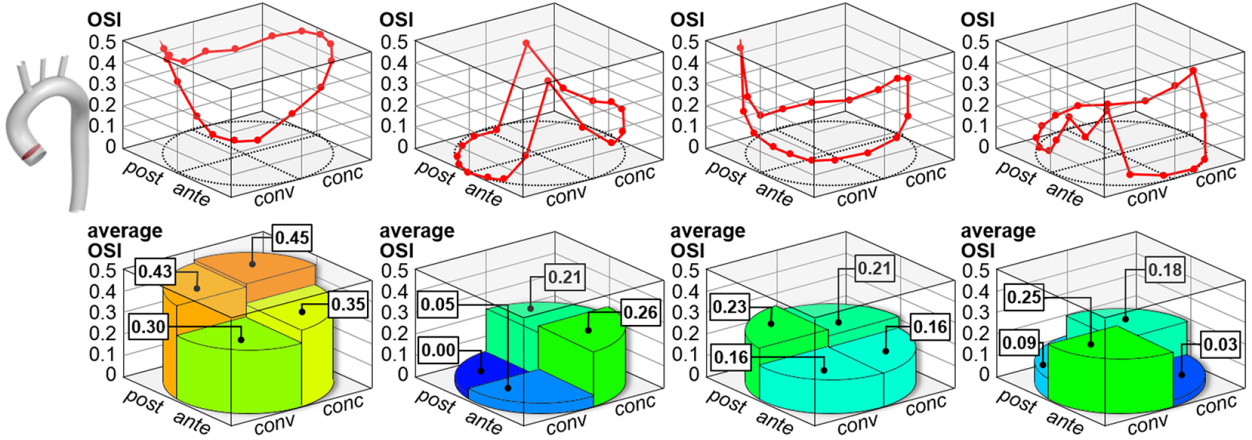

b

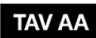

LR-BAV AA

RN-BAV AA

NL-BAV AA
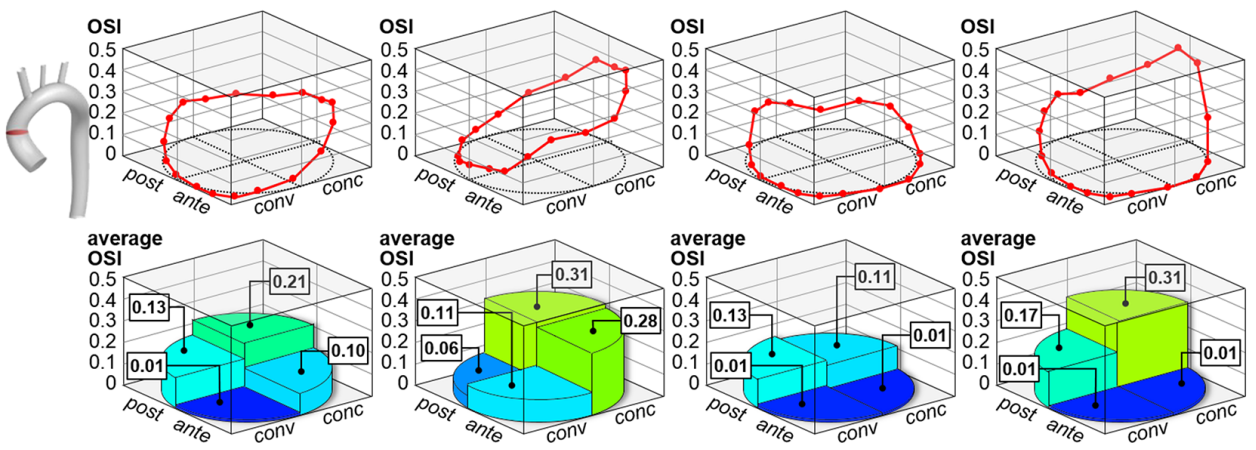

Figure 7. Local and quadrant-averaged OSI distributions in (a) the proximal section, and (b) the middle section of each model (post: posterior, ante: anterior, conv: convexity, conc: concavity). 
the aortic wall. In the middle AA section (Figure 7(b)), the circumferential OSI distributions exhibit less variability than in the proximal section. While the OSI variations between the four quadrants of the TAV AA remain qualitatively similar to those in the proximal section, they indicate greater unidirectionality (mean OSI: 0.11 in middle section vs. 0.38 in proximal section). In the LR-BAV AA, the bidirectionality of the flow observed in the concavity of the proximal aortic wall is maintained in the middle section, suggesting the weak effect of the wall curvature on WSS directionality in this model. The middle section of the RN-BAV AA experiences an overall decrease in WSS oscillation (mean OSI: 0.07) relative to the proximal section (mean OSI: 0.19). Lastly, the effect of the wall curvature is more apparent in the NL-BAV AA, as indicated by the migration of the unidirectional WSS from the posterior convexity and anterior concavity in the proximal section to the anterior convexity and concavity in the middle section.

\section{Discussion}

\subsection{BAV Morphotypes and Flow Abnormalities}

The models reveal the existence of notable flow abnormalities in BAV AAs relative to a normal TAV AA. Consistent with previous reports [8] [10] [20] [24], our FSI results indicate that, regardless of its morphotype, the BAV gives rise to a skewed helical flow in the AA, which contrasts with the smooth streamlined flow observed downstream of the TAV. Those flow abnormalities affect the WSS environment of the AA by altering its angular distribution along the wall circumference, its local magnitude and directionality. Consistent with previous 4D MRI measurements [8] [10], our simulations indicate that the TAV subjects the AA to a relatively uniform WSS distribution along the circumference, while the BAV generates strongly asymmetric WSS distributions, whose skewness depends on the type of cusp fusion and varies as the flow develops along the AA. Similarly, while all BAVs generate regions of WSS overload in the proximal AA relative to the TAV, the type of cusp fusion strongly affects the location of stress overload. In the middle AA, while the flow development along the curved aortic wall tends to normalize the WSS levels in the LR-BAV AA, it amplifies flow helicity in the RN-BAV and NL-BAV AAs, which results in sustained elevated WSS in those models. Those WSS abnormalities are exacerbated during the deceleration phase in the RN-BAV and NL-BAV AAs, as suggested by the extensive WSS overloads imposed on the middle and distal wall segments relative to the TAV and LR-BAV AAs.

\subsection{Comparison with PCMRI Measurements}

The WSS levels computed by our models are systematically higher than those previously reported in vivo. In the proximal AA, the maximum WSS predicted along the wall circumference at peak systole was 19.3 and 21.0 dyn $/ \mathrm{cm}^{2}$ in the TAV AA and LR-BAV AA, respectively. Those levels are above those captured with phasecontrast cardiac magnetic resonance measurements, which reported maximum peak-systolic WSS magnitudes of 8.5 and $15.6 \mathrm{dyn} / \mathrm{cm}^{2}$, respectively, in similar anatomies [25]. Similarly, in the middle AA, the circumferentially averaged WSS predicted in the TAV, LR-BAV and RN-BAV AAs at peak systole were 37.9, 34.5 and 32.5 $\mathrm{dyn} / \mathrm{cm}^{2}$, respectively. Those levels are one order-of-magnitude larger than those reported by PCMRI in the tubular AA portion of TAV and LR-BAV patients ( $4 \pm 1$ and $8 \pm 2$ dyn $/ \mathrm{cm}^{2}$, respectively) [26] and those reported by 4D flow MRI in TAV, LR-BAV and RN-BAV patients $\left(\sim 6 \mathrm{dyn} / \mathrm{cm}^{2}\right)$ [24]. The nature of those discrepancies is two-fold. First, our FSI simulations implemented a finer spatial resolution (near-wall spatial resolution: 100 $\mu \mathrm{m})$ than the MRI measurements (spatial resolution: $2 \mathrm{~mm}$ ). Second, as compared to in vivo measurements conducted in dilated BAV aortas, our models were based on a normal AA geometry, which permitted to isolate effectively the impact of the valve anatomy on AA hemodynamics while discarding the effects of a pre-existing aortopathy or the inherent variability in AA anatomy.

\subsection{Implications for BAV Aortopathy}

The flow predictions reported in this study support a possible role for hemodynamic stresses in the pathogenesis of BAV aortopathy. The stress abnormalities generated by the LR-BAV were concentrated in the proximal AA and consisted of a moderate WSS overload along the wall circumference, and the existence of a unidirectional WSS on the wall convexity and a bidirectional WSS on the wall concavity, which contrasted with the bidirectional WSS predicted along the TAV AA circumference. Interestingly, the dilation of the thoracic AA in LRBAV patients and the associated structural wall abnormalities localize primarily to the convexity of the AA wall 
[27] [28] and affect more predominantly the aortic root (i.e., type-1 dilation phenotype) and tubular AA portion (i.e., type-2 dilation phenotype) [19]. Collectively, those observations suggest that the unidirectional WSS environment on the convexity of the proximal LR-BAV AA could trigger a local injury response causing the progressive degradation of the aortic media. The sensitivity of the AA wall to such WSS environment and its dramatic downstream effects on extracellular matrix degeneration have been evidenced ex vivo [17], confirming the susceptibility of this unidirectional WSS to focally mediate aortic medial degradation.

The validity of this hemodynamic etiology is also supported by our flow predictions in the NL-BAV and RN-BAV AA models. The WSS abnormalities predicted in both models consisted of intense WSS overloads and contrasted WSS directionalities along the wall circumference. Interestingly, those WSS abnormalities were less local and more diffuse over the proximal and middle AA than in the LR-BAV AA, which correlates with the predominant type-3 dilation phenotype (i.e., dilation of both the entire AA and the transverse aortic arch) typically observed in those patients [19]. While causality between those WSS patterns and the local biology of the wall has not been established yet, the present study provides the mechanical data required for its future ex vivo investigation using the shear stress device designed in our laboratory [29].

\subsection{Limitations}

The flow conditions prescribed at the inlet of the aorta model are idealized as they were generated by extrapolating velocity profiles obtained from previously published 2D FSI simulations [17]. While this approach may seem simplistic, it captured most of the flow features observed by PCMRI in realistic patient-specific aorta anatomies [8] [20]. In this context, although caution should be taken before extrapolating the quantitative WSS data to human aortas, the analysis described in this paper provides new important qualitative insights into the flow and WSS differences between TAV and BAV AAs. Improved unified models integrating the 3D geometries of the valve and the AA are currently under development in our laboratory.

In addition, while in vivo and in vitro studies have demonstrated the existence of a transitional or slightly turbulent flow in the aorta, aortic flow was modeled as laminar in this study. Although this simplification may generate some inaccuracy at peak systole and in the early deceleration phase, it permitted to capture the near-native hemodynamic characteristics in the ascending aorta during most of the cardiac cycle. Improved models based on direct numerical simulations may be needed to resolve turbulence explicitly at the highest level of fidelity. However, this approach requires a computational grid able to resolve all the scales and is therefore not practical for FSI problems.

\section{Conclusion}

In this study, we quantified the flow and WSS environments in a normal, non-dilated AA subjected to the flows of a TAV and three BAV morphotypes. The results demonstrate: 1) the existence of abnormalities in WSS magnitude, directionality and circumferential distribution on BAV AAs; and 2) the dependence of the locations of WSS abnormalities on the type of BAV cusp fusion. This flow characterization sheds new light on the potential role played by hemodynamic stresses in BAV disease and provides the mechanical data required for the rigorous assessment of the hemodynamic theory of BAV aortopathy. Future implementation of those models with patient-specific anatomies and flow conditions may guide the clinical assessment of valvular function and the selection of appropriate treatment strategies.

\section{Acknowledgements}

This work was supported by the National Science Foundation under Grant CMMI-1148558; and the American Heart Association under Grant 14PRE18940010. The authors would like to thank Andrew McNally (University of Notre Dame) for his technical assistance with the model and Samantha Atkins (University of Notre Dame) for providing manuscript editing assistance.

\section{References}

[1] Ward, C. (2000) Clinical Significance of the Bicuspid Aortic Valve. Heart (British Cardiac Society), 83, 81-85. http://dx.doi.org/10.1136/heart.83.1.81

[2] Sabet, H.Y., Edwards, W.D., Tazelaar, H.D. and Daly, R.C. (1999) Congenitally Bicuspid Aortic Valves: A Surgical 
Pathology Study of 542 Cases (1991 through 1996) and a Literature Review of 2,715 Additional Cases. Mayo Clinic Proceedings, 74, 14-26. http://dx.doi.org/10.4065/74.1.14

[3] Sievers, H. and Schmidtke, C. (2007) A Classification System for the Bicuspid Aortic Valve from 304 Surgical Specimens. The Journal of Thoracic and Cardiovascular Surgery, 133, 1226-1233. http://dx.doi.org/10.1016/j.jtcvs.2007.01.039

[4] Nkomo, V.T., Enriquez-Sarano, M., Ammash, N.M., Melton 3rd, L.J., Bailey, K.R., Desjardins, V., et al. (2003) Bicuspid Aortic Valve Associated with Aortic Dilatation: A Community-Based Study. Arteriosclerosis, Thrombosis, and Vascular Biology, 23, 351-356. http://dx.doi.org/10.1161/01.ATV.0000055441.28842.0A

[5] Girdauskas, E., Borger, M.A., Secknus, M.A., Girdauskas, G. and Kuntze, T. (2011) Is Aortopathy in Bicuspid Aortic Valve Disease a Congenital Defect or a Result of Abnormal Hemodynamics? A Critical Reappraisal of a One-Sided Argument. European Journal of Cardio-Thoracic Surgery: Official Journal of the European Association for CardioThoracic Surgery, 39, 809-814. http://dx.doi.org/10.1016/j.ejcts.2011.01.001

[6] Atkins, S. and Sucosky, P. (2014) The Etiology of Bicuspid Aortic Valve Disease: Focus on Hemodynamics. World Journal of Cardiology, 12, 1227-1233. http://dx.doi.org/10.4330/wjc.v6.i12.1227

[7] Hope, M.D., Meadows, A.K., Hope, T.A., Ordovas, K.G., Reddy, G.P., Alley, M.T., et al. (2008) Images in Cardiovascular Medicine. Evaluation of Bicuspid Aortic Valve and Aortic Coarctation with 4D Flow Magnetic Resonance Imaging. Circulation, 117, 2818-2819. http://dx.doi.org/10.1161/CIRCULATIONAHA.107.760124

[8] Barker, A.J., Lanning, C. and Shandas, R. (2010) Quantification of Hemodynamic Wall Shear Stress in Patients with Bicuspid Aortic Valve Using Phase-Contrast MRI. Annals of Biomedical Engineering, 38, 788-800. http://dx.doi.org/10.1007/s10439-009-9854-3

[9] Bauer, M., Siniawski, H., Pasic, M., Schaumann, B. and Hetzer, R. (2006) Different Hemodynamic Stress of the Ascending Aorta Wall in Patients with Bicuspid and Tricuspid Aortic Valve. Journal of Cardiac Surgery, 21, 218-220. http://dx.doi.org/10.1111/j.1540-8191.2006.00219.x

[10] Bissell, M.M., Hess, A.T., Biasiolli, L., Glaze, S.J., Loudon, M., Pitcher, A., et al. (2013) Aortic Dilation in Bicuspid Aortic Valve Disease: Flow Pattern Is a Major Contributor and Differs with Valve Fusion Type. Circulation Cardiovascular Imaging, 6, 499-507. http://dx.doi.org/10.1161/CIRCIMAGING.113.000528

[11] Saikrishnan, N., Yap, C.-H., Milligan, N.C., Vasilyev, N.V and Yoganathan, A.P. (2012) In Vitro Characterization of Bicuspid Aortic Valve Hemodynamics Using Particle Image Velocimetry. Annals of Biomedical Engineering, 40, 1760-1775. http://dx.doi.org/10.1007/s10439-012-0527-2

[12] Seaman, C., Akingba, A. and Sucosky, P. (2014) Steady Flow Hemodynamic and Energy Loss Measurements in Normal and Simulated Calcified Tricuspid and Bicuspid Aortic Valves. Journal of Biomechanical Engineering, 136, Article ID: 041001. http://dx.doi.org/10.1115/1.4026575

[13] Seaman, C. and Sucosky, P. (2014) Anatomic versus Effective Orifice Area in a Bicuspid Aortic Valve. Echocardiography, 31, 1028. http://dx.doi.org/10.1111/echo.12720

[14] Chandra, S., Rajamannan, N.M. and Sucosky, P. (2012) Computational Assessment of Bicuspid Aortic Valve WallShear Stress: Implications for Calcific Aortic Valve Disease. Biomechanics and Modeling in Mechanobiology, 11, 1085-1096. http://dx.doi.org/10.1007/s10237-012-0375-x

[15] Nathan, D.P., Xu, C., Plappert, T., Desjardins, B., Gorman, J.H., Bavaria, J.E., et al. (2011) Increased Ascending Aortic Wall Stress in Patients with Bicuspid Aortic Valves. The Annals of Thoracic Surgery, 92, 1384-1389. http://dx.doi.org/10.1016/j.athoracsur.2011.04.118

[16] Pasta, S., Rinaudo, A., Luca, A., Pilato, M., Scardulla, C., Gleason, T.G., et al. (2013) Difference in Hemodynamic and Wall Stress of Ascending Thoracic Aortic Aneurysms with Bicuspid and Tricuspid Aortic Valve. Journal of Biomechanics, 46, 1729-1738. http://dx.doi.org/10.1016/j.jbiomech.2013.03.029

[17] Atkins, S.K., Cao, K., Rajamannan, N.M. and Sucosky, P. (2014) Bicuspid Aortic Valve Hemodynamics Induces Abnormal Medial Remodeling in the Convexity of Porcine Ascending Aortas. Biomechanics and Modeling in Mechanobiology, 13, 1209-1225. http://dx.doi.org/10.1007/s10237-014-0567-7

[18] Fazel, S.S., Mallidi, H.R., Lee, R.S., Sheehan, M.P., Liang, D., Fleischman, D., et al. (2008) The Aortopathy of Bicuspid Aortic Valve Disease Has Distinctive Patterns and Usually Involves the Transverse Aortic Arch. The Journal of Thoracic and Cardiovascular Surgery, 135, 901-907, 907.e1-2.

[19] Kang, J.-W., Song, H.G., Yang, D.H., Baek, S., Kim, D.-H., Song, J.-M., et al. (2013) Association between Bicuspid Aortic Valve Phenotype and Patterns of Valvular Dysfunction and Bicuspid Aortopathy: Comprehensive Evaluation Using MDCT and Echocardiography. JACC Cardiovascular Imaging, 6, 150-161. http://dx.doi.org/10.1016/j.jcmg.2012.11.007

[20] Hope, M.D., Hope, T.A., Meadows, A.K., Ordovas, K.G., Urbania, T.H., Alley, M.T., et al. (2010) Bicuspid Aortic Valve: Four-Dimensional MR Evaluation of Ascending Aortic Systolic Flow Patterns. Radiology, 255, 53-61. 
http://dx.doi.org/10.1148/radiol.09091437

[21] Olufsen, M.S., Peskin, C.S., Kim, W.Y., Pedersen, E.M., Nadim, A. and Larsen, J. (2000) Numerical Simulation and Experimental Validation of Blood Flow in Arteries with Structured-Tree Outflow Conditions. Annals of Biomedical Engineering, 28, 1281-1299. http://dx.doi.org/10.1114/1.1326031

[22] Lantz, J., Renner, J. and Karlsson, M. (2011) Wall Shear Stress in a Subject Specific Human Aorta-Influence of Fluid-Structure Interaction. International Journal of Applied Mechanics, 3, 759-778. http://dx.doi.org/10.1142/S1758825111001226

[23] Donea, J., Guiliani, S. and Halleux, J.P. (1982) An Arbitrary Lagrangian-Eulerian Finite-Element Method for Transient Dynamic Fluid Structure Interactions. Computer Methods in Applied Mechanics and Engineering, 33, 689-723. http://dx.doi.org/10.1016/0045-7825(82)90128-1

[24] Mahadevia, R., Barker, A.J., Schnell, S., Entezari, P., Kansal, P., Fedak, P.W.M., et al. (2014) Bicuspid Aortic Cusp Fusion Morphology Alters Aortic 3D Outflow Patterns, Wall Shear Stress and Expression of Aortopathy. Circulation, 129, 673-682. http://dx.doi.org/10.1161/CIRCULATIONAHA.113.003026

[25] Hope, M.D., Hope, T.A., Crook, S.E.S., Ordovas, K.G., Urbania, T.H., Alley, M.T., et al. (2011) 4D Flow CMR in Assessment of Valve-Related Ascending Aortic Disease. JACC Cardiovascular Imaging, 4, 781-787. http://dx.doi.org/10.1016/j.jcmg.2011.05.004

[26] Barker, A.J., Markl, M., Bürk, J., Lorenz, R., Bock, J., Bauer, S., et al. (2012) Bicuspid Aortic Valve Is Associated with Altered Wall Shear Stress in the Ascending Aorta. Circulation Cardiovascular Imaging, 5, 457-466. http://dx.doi.org/10.1161/CIRCIMAGING.112.973370

[27] Cotrufo, M., Della Corte, A., De Santo, L.S., Quarto, C., De Feo, M., Romano, G., et al. (2005) Different Patterns of Extracellular Matrix Protein Expression in the Convexity and the Concavity of the Dilated Aorta with Bicuspid Aortic Valve: Preliminary Results. The Journal of Thoracic and Cardiovascular Surgery, 130, 504-511. http://dx.doi.org/10.1016/j.jtcvs.2005.01.016

[28] Della Corte, A., Quarto, C., Bancone, C., Castaldo, C., Di Meglio, F., Nurzynska, D., et al. (2008) Spatiotemporal Patterns of Smooth Muscle Cell Changes in Ascending Aortic Dilatation with Bicuspid and Tricuspid Aortic Valve Stenosis: Focus on Cell-Matrix Signaling. The Journal of Thoracic and Cardiovascular Surgery, 135, 8-18.e2. http://dx.doi.org/10.1016/j.jtcvs.2007.09.009

[29] Sucosky, P., Padala, M., Elhammali, A., Balachandran, K., Jo, H. and Yoganathan, A.P. (2008) Design of an ex Vivo Culture System to Investigate the Effects of Shear Stress on Cardiovascular Tissue. Journal of Biomechanical Engineering, 130, 35001-35008. http://dx.doi.org/10.1115/1.2907753 